\title{
On the existence of birational maximal Cohen-Macaulay modules over biradical extensions in mixed characteristic
}

\author{
Prashanth Sridhar* \\ University of Kansas \\ 405 Snow Hall, 1460 Jayhawk Blvd, \\ Lawrence, Kansas, USA 66045 \\ e-mail: prashanth@ku.edu
}

\begin{abstract}
Let $S$ be an unramified regular local ring of mixed characteristic $p \geq 3$ and $S^{p}$ the subring of $S$ obtained by lifting to $S$ the image of the Frobenius map on $S / p S$. Let $R$ be the integral closure of $S$ in a biradical extension of degree $p^{2}$ of its quotient field obtained by adjoining $p$-th roots of sufficiently general square free elements $f, g \in S^{p}$. We show that $R$ admits a birational maximal Cohen-Macaulay module. It is noted that $R$ is not automatically Cohen-Macaulay.

Keywords: birational maximal Cohen-Macaulay module, biradical extension, mixed characteristic, unramified regular local ring, integral closure.

Classification Codes: 13B22, 13C10, 13C14, 13C15, 13D22, 13E05, $13 \mathrm{H} 05$.
\end{abstract}

\section{Introduction}

The existence of maximal Cohen-Macaulay (MCM) modules (2.1) over catenary rings is largely an open problem. Hochster conjectured that every complete local domain admits an MCM module (see for example [Hoc73] and [Rob12]), but this is known to be true only in very few cases. The primary goal of this article is to show the existence of an MCM module for a new class of rings.

Hochster's conjecture reduces to the integral closure of a complete regular local ring in a normal extension of its fraction field, so that one may approach the problem from this viewpoint. Let $S$ denote an unramified regular local ring with quotient field $L$ and

${ }^{*}$ Corresponding Author 
$R$ the integral closure of $S$ in a finite field extension $K / L$. In Rob80 it is shown that if $K / L$ is Abelian and the degree of the extension is not divisible by the characteristic of the residue field, then $R$ is Cohen-Macaulay. Note that this applies when $S$ contains the rational numbers. The proof uses the fact that the group algebra $k[G]$ is a product of fields when the residue field $k$ of $S$ is algebraically closed and $G=\operatorname{Gal}(K / L)$. There is no direct analog of the argument when $\operatorname{char}(k)$ divides the order of $G$. In fact, the conclusion fails in mixed characteristic as shown in [Koh86] and [Kat99].

Motivated by the above phenomenon and the studies in [Kat99, Kat21 and [Sri21, we consider extensions $K / L$ with the property that $R$ is $S$-free when $S$ contains a field but not necessarily so otherwise. Kummer theory tells us that Abelian extensions of a field of characteristic zero containing "suitable" roots of unity are repeated radical extensions. Thus it is natural to study repeated $n$-th root extensions of an unramified regular local ring of mixed characteristic $p>0$ with the property that $p \mid n$. If $S$ were to contain the rational numbers or if $S$ were of mixed characteristic $p>0$ and $p \nmid n$, then it follows that the integral closure of $S$ in an arbitrary repeated radical extension is Cohen-Macaulay, see [Sri21] and HK19.

Now assume additionally that $S$ has mixed characteristic $p \geq 3$. If $K$ is the extension by a $p$-th root of a square free element of $S$, then $R$ is Cohen-Macaulay as shown in Kat99. In contrast, we shall see that the integral closure $R$ fails to be Cohen-Macaulay in a finite square free tower of $p$-th roots. In this paper, we consider the existence of maximal CohenMacaulay modules over the integral closure of $S$ in an extension of degree $p^{2}$, obtained by adjoining $p$-th roots of sufficiently general square free elements. The generality and square free conditions on the elements are natural, since any given multi-radical extension can be embedded in a sufficiently large, general, square free tower.

Let $S^{p}$ denote the subring of $S$ obtained by lifting the image of the Frobenius map on $S / p S$ to $S$. Towards the above goal, the immediate obstruction is when $f, g \in S^{p}$, see KS21]. We now outline our principal findings. Fix $f, g \in S^{p}$ square free, non-units that form a regular sequence in $S$ or units that are not $p$-th powers in $S$. Let $\omega^{p}=f$ and $\mu^{p}=g$. If $f, g$ are units, assume further that $[L(\omega, \mu): L]=p^{2}$. Given integers $n, k \geq 1$, let $S^{p^{k} \wedge p^{n}} \subset S$ be the multiplicative subset of $S$ consisting of elements expressible in the form $x^{p^{k}}+y \cdot p^{n}$ for some $x, y \in S$. For a discussion of the case $p=2$, see [Sri21], where the results are sharper since in that case such extensions are automatically Abelian. When $p \geq 3$, the presence of a $p$-th root of unity in $S$ necessarily ramifies $p$, so we do not quite have the same leg room. The main result of this paper is

Theorem. 4.10 Let $(S, \mathfrak{m})$ be an unramified regular local ring of mixed characteristic $p \geq 3$. Then

\section{1. $R$ is Cohen-Macaulay if}

(a) At least one of $S[\omega], S[\mu]$ is not integrally closed. 
(b) $S[\omega], S[\mu]$ are integrally closed and $f g^{i} \notin S^{p \wedge p^{2}}$ for all $1 \leq i \leq p-1$.

2. Let $S[\omega], S[\mu]$ be integrally closed such that $f g \in S^{p \wedge p^{2}}$. Then $R$ is Cohen-Macaulay if and only if $Q:=(p, f, g) \subset S$ is a two generated ideal or all of $S$. Moreover, $p . d \cdot S(R) \leq 1$ and $\nu_{S}(R) \leq p^{2}+1$.

3. If $Q:=(p, f, g) \subseteq S$ has grade three, $R$ admits a birational maximal Cohen-Macaulay module.

In section 2, we set up convention and make some preliminary remarks that will be used subsequently. In section 3 , we provide some sufficient conditions for $R$ to be CohenMacaulay by showing a more general version of 4.10(1), see 3.1 and 3.3 . We also identify the conductor of $R$ to the complete intersection $\operatorname{ring} A:=S[\omega, \mu]$ in a crucial case. In section 4, we prove parts (2) and (3) of 4.10. We see that $R$ is not "too far" from being Cohen-Macaulay, in the sense that it can be generated by $p^{2}+1$ elements over the base ring $S$ and $p . d \cdot S(R) \leq 1$. However, it is not as close as it appears, since if $\operatorname{dim}(S) \geq 3$ it could be that $R$ does not even satisfy Serre's condition $S_{3}$. We then show that $R$ admits a birational maximal Cohen-Macaulay module when $Q:=\left(p, h_{1}, h_{2}\right)$ has grade three. The condition on $Q$ can be viewed as a further generality condition on the chosen elements. For an $A$-module $M$, let $M^{*}=\operatorname{Hom}_{A}(M, A)$ be the dual module. The strategy here is to realize $R=I^{*}$ for a suitable ideal $I \subseteq A$ and identifying an ideal $J \subseteq A$ such that $J^{*}$ is an $I^{*}$ module and $J^{*}$ is $S$-free.

\section{Preliminaries}

All rings considered are commutative and Noetherian and all modules finitely generated.

\section{Convention 2.1.}

1. Let $R$ be a Noetherian ring and $M$ an $R$-module. For $G \subseteq M$ a subset, the notation $M=\langle G\rangle_{R}$ means that $M$ is generated as a $R$-module by $G$.

2. Let $(R, \mathfrak{m})$ be a local ring of dimension $d$. A nonzero $R$-module $M$ is a maximal Cohen-Macaulay module (MCM) over $R$ if it is finitely generated and every (some) system of parameters of $R$ is a regular sequence on $M$. If $R$ is an arbitrary Noetherian ring, then an $R$-module $M$ is a maximal Cohen-Macaulay module if for all maximal ideals $\mathfrak{m} \subseteq R, M_{\mathfrak{m}}$ is an MCM module over $R_{\mathfrak{m}}$.

3. Let $R$ be a domain and $M$ an $R$-module. Denote by $M_{R}^{*}$, the dual module $\operatorname{Hom}_{R}(M, R)$. If $R$ is clear from the context, denote it by $M^{*}$. Suppose that $M \subseteq K$, where $K$ denotes the field of fractions of $R$. Then via the identification $\operatorname{Hom}_{R}(M, R) \simeq(R: K M)$, we use $M^{*}$ to denote $\left(R:_{K} M\right)$ as well. 
4. If $R$ is a Noetherian ring of dimension at least one, denote

$$
N N L_{1}(R):=\left\{P \in \operatorname{Spec}(R) \mid \operatorname{height}(P)=1, R_{P} \text { is not a DVR }\right\}
$$

5. Suppose $S$ is a ring and $p \in \mathbb{Z}$ is such that $p \in S$ is a non-unit. Let $F: S / p S \rightarrow S / p S$ be the Frobenius map. Let $S^{p}$ denote the subring of $S$ obtained by lifting the image of $F$ to $S$. Let $S^{p^{k} \wedge p^{n}}$ for $k, n \geq 1$ denote the multiplicative subset of $S$ of elements expressible in the form $x^{p^{k}}+y p^{n}$ for some $x, y \in S$. In particular, $S^{p \wedge p}=S^{p}$.

6. For a local ring $R$ and an $R$-module $M$, denote $\nu_{R}(M)$ for the minimal number of generators of $M$ over $R$.

Remark 2.2. Let $S \subseteq C \subseteq D$ be an extension of Noetherian domains such that $S$ is integrally closed, $D$ is module finite over $S$ and $D$ is birational to $C$. Then if $C$ is regular in codimension one, so is $D$. (see [Vas91] [Theorem 2.4] for example)

Remark 2.3. Let $S \subseteq D$ be an extension of Noetherian domains such that going down holds. Let $\bar{D}$ denote the integral closure of $D$ in its field of fractions $K$ and assume $\bar{D}$ is finite over $D$. If $c \cdot u, c \cdot v \in D:_{K} \bar{D}$ with $c \in D$ and $u . v \in S$ such that there exists no height one prime of $S$ containing both of them, then $N N L_{1}(D) \subseteq V(c)$.

Remark 2.4. Let $A$ be a Noetherian Gorenstein local domain and $R$ the integral closure of $A$. Assume that $R$ is module finite over $A$. Then for every height one unmixed ideal $I \subseteq A, I^{*}$ is Cohen-Macaulay if and only if $A / I$ is Cohen-Macaulay (see Sri21][Proposition 2.11] for example). In particular, $R$ is Cohen-Macaulay if and only if the conductor of $R$ to $A$ is Cohen-Macaulay.

Remark 2.5. Let $S \subseteq R$ be a finite extension of local rings such that $S$ is Gorenstein and $R$ is Cohen-Macaulay. Then for an $R$-module $M$, we have $\operatorname{Hom}_{R}\left(M, \omega_{R}\right) \simeq \operatorname{Hom}_{S}(M, S)$ as $R$-modules (and $S$-modules), where $\omega_{R}$ is the canonical module of $R$.

Convention 2.6. We will assume the following notation for the rest of the paper unless otherwise specified. Let $S$ denote a Noetherian, integrally closed domain of dimension $d$ and $L$ its field of fractions. Assume $\operatorname{Char}(L)=0$. Fix $3 \leq p \in \mathbb{Z}$ a prime and assume that $p \in S$ is a principal prime such that $S / p S$ is integrally closed. Note here that an unramified regular local ring of mixed characteristic $p$ satisfies the above hypothesis, though not all results here require this specific setting.

Say that a subset $E \subset S$ satisfies $\mathscr{A}_{1}$, if for all distinct $x, y \in E$, there exists no height one prime $Q \subset S$ such that $(x, y) \subset Q$. An element $c \in S$ is square free if for all height one primes $P \subset S$ containing $c, P S_{P}=(c) S_{P}$.

Fix $f, g \in S^{p}$ square free, non-units, satisfying $\mathscr{A}_{1}$ or $f, g \in S^{p}$ units such that they are not $p$-th powers in $S$. Write $f=h_{1}^{p}+a \cdot p$ and $g=h_{2}^{p}+b \cdot p$ with $h_{1}, h_{2}, a, b \in S$. 
Let $W, U$ be indeterminates over $S$. We have the monic irreducible polynomials $F(W):=$ $W^{p}-f \in S[W]$ and $G(U):=U^{p}-g \in S[U]$. Let $\omega, \mu$ be roots of $F(W)$ and $G(U)$ respectively and set $K:=L(\omega, \mu)$. Assume that $G(U)$ is irreducible over $L(\omega)$, so that $[K$ : $L]=p^{2}$. If $S$ is a unique factorization domain and $f, g$ are non units, it can be shown that $[K: L]=p^{2}$ is automatic. Let $R$ be the integral closure of $S$ in $K$, that is $R$ is the integral closure of $A:=S[\omega, \mu]$. Note that, $A \simeq S[W, U] /(F(W), G(U)), S[\omega] \simeq S[W] /(F(W))$ and $S[\mu] \simeq S[U] /(G(U))$.

Remark 2.7. From [Sri21] [Proposition 2.10], if exactly one of $f, g$ lies in $S^{p}$ then $R$ is $S$-free. Moreover Sri21][Example 2.12] shows that $R$ need not be $S$-free when $f, g \notin S^{p}$. However, to construct an MCM module over $R$ it suffices to consider the case $f, g \in S^{p}$ when $S$ is a complete unramified regular local ring with perfect residue field, see [KS21. This motivates us to understand the case $f, g \in S^{p}$.

Lemma 2.8 ([Kat99]). Let $p=2 k+1$ and $h \in S \backslash p S$. Let $W$ be an indeterminate over $S$. If

$$
C:=\left(W^{p}-h^{p}\right)-(W-h)^{p}=\sum_{j=1}^{k}(-1)^{j+1}\left(\begin{array}{l}
p \\
j
\end{array}\right)(W \cdot h)^{j}\left[W^{p-2 j}-h^{p-2 j}\right]
$$

$C^{\prime}:=(p(W-h))^{-1} \cdot C$ and $\tilde{P}:=(p, W-h) S[W]$, then $C^{\prime} \notin \tilde{P}$.

Lemma 2.9. Let $p=2 k+1$ and $h \in S \backslash p S$. Let $W$ be an indeterminate over $S$. Suppose $C^{\prime}$ is as defined in 2.8. Then $C^{\prime} \equiv h^{p-1} \bmod (p, W-h) S[W]$.

Proof. We have in $S[W]$

$$
\begin{aligned}
C^{\prime} & =\sum_{j=1}^{k}(-1)^{j+1} j^{-1}\left(\begin{array}{c}
p-1 \\
j-1
\end{array}\right)(W \cdot h)^{j}\left[W^{p-2 j-1}+\cdots+h^{p-2 j-1}\right] \\
& \equiv \sum_{j=1}^{k}(-1)^{j+1} j^{-1}\left(\begin{array}{c}
p-1 \\
j-1
\end{array}\right) h^{2 j} \cdot(p-2 j) \cdot h^{p-2 j-1} \bmod (p, W-h) \\
& \equiv-2 h^{p-1} \sum_{j=1}^{k}(-1)^{j+1}\left(\begin{array}{c}
p-1 \\
j-1
\end{array}\right) \bmod (p, W-h) \\
& \equiv-h^{p-1}(-1)^{k+1}\left(\begin{array}{c}
2 k \\
k
\end{array}\right) \bmod (p, W-h) \\
& \equiv h^{p-1} \bmod (p, W-h)
\end{aligned}
$$


Convention 2.10. Suppose $h_{1}, h_{2} \in S \backslash p S$. In this case, let $C_{1}^{\prime}$ and $C_{2}^{\prime}$ denote respectively the elements in the rings $S[W]$ and $S[U]$ obtained by setting $h=h_{1}$ and $h=h_{2}$ in 2.8 , Denote by $c_{1}^{\prime}$ and $c_{2}^{\prime}$ their respective images in the rings $S[\omega]$ and $S[\mu]$ respectively. If $h_{1}=0$ $\left(h_{2}=0\right)$, simply set $c_{1}^{\prime}=0\left(c_{2}^{\prime}=0\right)$. Denote by $d_{i}$ the corresponding element in $S\left[\omega \mu^{i}\right]$ for $1 \leq i \leq p-1$.

Proposition 2.11 ([Kat99]). With notation as specified above, $S[\omega]$ is integrally closed if and only if $f \notin S^{p \wedge p^{2}}$. Further, if $S[\omega]$ is not integrally closed, write $f=h_{1}^{p}+a^{\prime} \cdot p^{2}$ for some $a^{\prime} \in S$. Then

(a) $\overline{S[\omega]}=\left(P^{*}\right)_{S[\omega]}=S\left[\omega, \tau_{1}\right]$ where $\tau_{1}=p^{-1} \cdot\left(\omega^{p-1}+h_{1} \omega^{p-2}+\cdots+h_{1}^{p-1}\right)$ and $P:=$ $\left(p, \omega-h_{1}\right) S[\omega]$.

(b) There are exactly two height one primes in $\overline{S[\omega]}$ containing $p$, namely $P:=(p, \omega-$ $\left.h_{1}, \tau_{1}\right) \overline{S[\omega]}$ and $Q:=\left(p, \omega-h_{1}, \tau-c_{1}^{\prime}\right) \overline{S[\omega]}$. Further, $P_{P}=\left(\omega-h_{1}\right)_{P}$ and $Q_{Q}=(p)_{Q}$.

(c) The element $\tau_{1}$ satisfies $l(T):=T^{2}-c_{1}^{\prime} T-a^{\prime} \cdot\left(\omega-h_{1}\right)^{p-2} \in S[\omega][T]$.

(d) $\overline{S[\omega]}=\left\langle 1, \omega, \omega^{2}, \ldots, \omega^{p-2}, \tau_{1}\right\rangle_{S}$ is $S$-free.

\section{Cohen-Macaulay integral closures}

In this section we identify scenarios where $R$ is $S$-free by showing more general versions of $1(a)$ and $1(b)$ in Theorem 4.10. We maintain notation as set up in 2.6.

Proposition 3.1. $R$ is $S$-free if at least one of the rings $S[\omega], S[\mu]$ is not integrally closed.

Proof. We organize the proof as follows:

1. Assume $S[\omega]$ and $S[\mu]$ are both not integrally closed. We then

(a) Identify a finite birational overring $A \hookrightarrow \mathscr{R} A$ such that $\mathscr{R} A$ satisfies $R_{1}$.

(b) Identify a "natural" finite birational overring $\mathscr{R} A \hookrightarrow Z$ such that $Z$ is $S$-free, so that $R=Z$ is $S$-free.

2. Assume exactly one of the rings $S[\omega], S[\mu]$ is integrally closed. We then take an identical path as indicated in (1) above.

1. (a) From 2.11, $f, g \in S^{p \wedge p^{2}}$. Write $f=h_{1}^{p}+a^{\prime} \cdot p^{2}$ and $g=h_{2}^{p}+b^{\prime} \cdot p^{2}$ for some $a^{\prime}, b^{\prime} \in S$. Note that $h_{1}, h_{2} \neq 0$ since $f, g$ are square free. We have from 2.11 that $S\left[\omega, \tau_{1}\right], S\left[\mu, \tau_{2}\right]$ are the respective normalizations of $S[\omega]$ and $S[\mu]$ where

$$
\tau_{1}=p^{-1} \cdot\left(\omega^{p-1}+h_{1} \omega^{p-2}+\cdots+h_{1}^{p-1}\right)
$$




$$
\tau_{2}=p^{-1} \cdot\left(\mu^{p-1}+h_{2} \mu^{p-2}+\cdots+h_{2}^{p-1}\right)
$$

Set $E:=A\left[\tau_{1}, \tau_{2}\right]$. Let $X, Y$ be indeterminates over $A$ and let $\phi: A[X, Y] \rightarrow E$ be the projection map sending $X \rightarrow \tau_{1}$ and $Y \rightarrow \tau_{2}$. From 2.11,

$$
X^{2}-c_{1}^{\prime} X-a^{\prime}\left(\omega-h_{1}\right)^{p-2}, Y^{2}-c_{2}^{\prime} Y-b^{\prime}\left(\mu-h_{2}\right)^{p-2} \in \operatorname{Ker}(\phi)
$$

Height one primes in $E$ containing $p$ correspond to height three primes in $A[X, Y]$ containing $\operatorname{Ker}(\phi)$ and $p$. Since $P:=\left(p, \omega-h_{1}, \mu-h_{2}\right)$ is the unique height one prime in $A$ containing $p$, any such height three prime in $A[X, Y]$ has to contain either $X$ or $X-c_{1}^{\prime}$. Likewise it contains either $Y$ or $Y-c_{2}^{\prime}$. Therefore if $Q \subseteq A[X, Y]$ is a height three prime containing $p$ and $\operatorname{Ker}(\phi)$, it must be that $\left(p, \omega-h_{1}, \mu-h_{2}, X-m, Y-n\right) \subseteq Q$ for some $m, n \in A$ and hence the containment must be an equality. Moreover, there is at least one height one prime in $E$ containing $p$, since $p$ is not a unit in $S$. Therefore, the only possibilities for height one primes in $E$ containing $p$ are

$$
\begin{gathered}
P_{1}:=\left(p, \omega-h_{1}, \mu-h_{2}, \tau_{1}, \tau_{2}\right) \\
P_{2}:=\left(p, \omega-h_{1}, \mu-h_{2}, \tau_{1}, \tau_{2}-c_{2}^{\prime}\right) \\
P_{3}:=\left(p, \omega-h_{1}, \mu-h_{2}, \tau_{1}-c_{1}^{\prime}, \tau_{2}\right) \\
P_{4}:=\left(p, \omega-h_{1}, \mu-h_{2}, \tau_{1}-c_{1}^{\prime}, \tau_{2}-c_{2}^{\prime}\right)
\end{gathered}
$$

We have $\omega \cdot F^{\prime}(\omega)=p \cdot f \in\left(S\left[\mu, \tau_{2}, \omega\right]:_{K} R\right)$ and identically $p \cdot g \in\left(S\left[\omega, \tau_{1}, \mu\right]:_{K} R\right)$ (see for example [HS06][Theorem 12.1.1]) and hence $p \cdot f, p \cdot g \in\left(E:_{K} R\right)$. From 2.3, $N N L_{1}(E) \subseteq V(p)$. But the localizations of $E$ at $P_{2}, P_{3}$ and $P_{4}$ are regular with uniformizing parameters being the images of $\omega-h_{1}, \mu-h_{2}$ and $p$ respectively. For example, consider $P_{2 P_{2}}$. Let $Q_{1}:=\left(p, \omega-h_{1}, \tau_{1}\right) S\left[\omega, \tau_{1}\right]$ and $Q_{2}:=\left(p, \mu-h_{2}, \tau_{2}-c_{2}^{\prime}\right) S\left[\mu, \tau_{2}\right]$. From 2.11, $Q_{1 Q_{1}}=\left(\omega-h_{1}\right)_{Q_{1}}$ and $Q_{2 Q_{2}}=(p)_{Q_{2}}$. Thus $P_{2 P_{2}}=\left(\omega-h_{1}\right)_{P_{2}}$. The $P_{3}$ and $P_{4}$ cases are similar. Note that however $P_{1 P_{1}}=\left(\omega-h_{1}, \mu-h_{2}\right)_{P_{1}}$.

Set $\eta_{1}=p^{-1}\left(\omega-h_{1}\right)\left(\mu-h_{2}\right)^{p-2} \in K$. Let $X$ be an indeterminate over $E$. Then $\eta_{1}$ satisfies $l(X) \in E[X]$ where $l(X):=X^{p-1}-\left(\tau_{1}-c_{1}^{\prime}\right)\left(\tau_{2}-c_{2}^{\prime}\right)^{p-2}$ since $p \cdot \tau_{1}=\left(\omega-h_{1}\right)^{p-1}+p \cdot c_{1}^{\prime}$ (similarly for $\left.p \cdot \tau_{2}\right)$. We claim that $\mathscr{R} A:=E\left[\eta_{1}\right]$ is regular in codimension one. From 2.2 , $N N L_{1}(\mathscr{R} A) \subseteq V\left(P_{1} \mathscr{R} A\right)$. Denote by $\overline{l(X)}$ the image of $l(X)$ in $\left(E / P_{1} E\right)[X]$. From 2.9 we have $c_{1}^{\prime} \equiv h_{1}^{p-1}$ and $c_{2}^{\prime} \equiv h_{2}^{p-1}$ in the ring $E / P_{1} E$, and thus 1

$$
\overline{l(X)}=X^{p-1}-\left(h_{1} h_{2}^{p-2}\right)^{p-1}=\prod_{k=1}^{p-1}\left(X+k h_{1} h_{2}^{p-2}\right) \in\left(E / P_{1} E\right)[X]
$$

\footnotetext{
${ }^{1}$ If $R$ is a ring of characteristic $p$ and $X, Y$ indeterminates over $R$, then for $X^{p-1}-Y^{p-1} \in R[X, Y]$, $X^{p-1}-Y^{p-1}=\prod_{i=1}^{p-1}(X+i Y)$.
} 
Thus, the only possibilities for height one primes in $\mathscr{R} A$ lying over $P_{1}$ are

$$
Q_{k}=\left(p, \omega-h_{1}, \mu-h_{2}, \tau_{1}, \tau_{2}, \eta_{1}+k h_{1} h_{2}^{p-2}\right) \mathscr{R} A
$$

for $1 \leq k \leq p-1$ and we have

$$
Q_{k Q_{k}}=\left(\omega-h_{1}, \mu-h_{2}, \eta_{1}+k h_{1} h_{2}^{p-2}\right)_{Q_{k}}
$$

Since $\left(\mu-h_{2}\right) \eta_{1}=\left(\tau_{2}-c_{2}^{\prime}\right)\left(\omega-h_{1}\right)$ and

$$
\eta_{1}, \tau_{2}-c_{2}^{\prime}, \prod_{j=1, j \neq k}^{p-1}\left(\eta_{1}+j h_{1} h_{2}^{p-2}\right) \notin Q_{k}
$$

we have $Q_{k Q_{k}}=\left(\mu-h_{2}\right)_{Q_{k}}=\left(\omega-h_{1}\right)_{Q_{k}}$. Therefore $\mathscr{R} A$ is regular in codimension one.

(b) Set $Z:=\langle T\rangle_{S}$ where $T:=T_{1} \cup T_{2} \cup T_{3}$ and

$$
\begin{gathered}
T_{1}:=\left\{\left(\omega-h_{1}\right)^{i}\left(\mu-h_{2}\right)^{j} \mid 0 \leq i, j \leq p-1, i+j<p-1\right\} \\
T_{2}:=\left\{p^{-1}\left(\omega-h_{1}\right)^{i}\left(\mu-h_{2}\right)^{j} \mid 0 \leq i, j \leq p-1, i+j \geq p-1, i+j \neq 2 p-2\right\} \\
T_{3}:=\left\{p^{-2}\left(\omega-h_{1}\right)^{p-1}\left(\mu-h_{2}\right)^{p-1}\right\}
\end{gathered}
$$

For every choice of $0 \leq i, j \leq p-1$, there is a unique element in $T$ with "leading coefficient" $\omega^{i} \mu^{j}$. Therefore the order of $T$ is $p^{2}$. Moreover, since $A$ is $S$-free with a basis given by $D:=\left\{\left(\omega-h_{1}\right)^{i}\left(\mu-h_{2}\right)^{j} \mid 0 \leq i, j \leq p-1\right\}$, the elements of $T$ are linearly independent over $S$. From the relations

$$
\begin{aligned}
& \left(\omega-h_{1}\right)^{p}=a^{\prime} p^{2}-p c_{1}^{\prime}\left(\omega-h_{1}\right) \\
& \left(\mu-h_{2}\right)^{p}=b^{\prime} p^{2}-p c_{2}^{\prime}\left(\mu-h_{2}\right)
\end{aligned}
$$

we get that $Z$ is a ring. Since $Z$ satisfies $S_{2}$, if we show $\mathscr{R} A \subseteq Z$, then from $2.2 Z=R$. Since $D \subseteq T$, we have $A \subseteq Z$. Since $\eta_{1}=p^{-1}\left(\omega-h_{1}\right)\left(\mu-h_{2}\right)^{p-2} \in T$, it only remains to be seen that $\tau_{1}, \tau_{2} \in Z$. But this is clear from the relation $\tau_{1}=p^{-1}\left(\omega-h_{1}\right)^{p-1}+c_{1}^{\prime}$ (analogously for $\tau_{2}$ ). Thus $Z=R$ and $R$ is $S$-free.

2. (a) Assume without loss of generality $\overline{S[\omega]}=S[\omega, \tau]$ where $\tau=p^{-1}\left(\omega^{p-1}+\cdots+h_{1}^{p-1}\right)$ and that $S[\mu]$ is integrally closed. Notice that if $P:=\left(p, \mu-h_{2}\right) \subseteq S[\mu]$ is the unique height one prime in $S[\mu]$ containing $p$, then $P_{P}=\left(\mu-h_{2}\right)_{P}$ since $\left(\mu-h_{2}\right)\left(\mu^{p-1}+\cdots+h_{2}^{p-1}\right)=b p$ and $b \notin p S$. From 2.11, $f \in S^{p \wedge p^{2}}$, so write $f=h_{1}^{p}+a^{\prime} p^{2}$.

Set $E:=S[\omega, \mu, \tau]$. From 2.11, it follows that there are precisely two height one primes in $E$ containing $p$, namely $P_{1}:=\left(p, \omega-h_{1}, \tau, \mu-h_{2}\right)$ and $P_{2}:=\left(p, \omega-h_{1}, \tau-c_{1}^{\prime}, \mu-h_{2}\right)$. From 2.3. $E$ is regular in codimension one outside of $P_{1}$ and $P_{2}$. It follows from 2.11(b) that $P_{1 P_{1}}=\left(\omega-h_{1}, \mu-h_{2}\right)_{P_{1}}$ and $P_{2 P_{2}}=\left(p, \mu-h_{2}\right)_{P_{2}}=\left(\mu-h_{2}\right)_{P_{2}}$. 
Set $\eta_{1}:=p^{-1}\left(\omega-h_{1}\right)\left(\mu-h_{2}\right)^{p-1} \in K . \eta_{1} \in R$ since it satisfies

$$
l(X):=X^{p-1}-\left(\tau-c_{1}^{\prime}\right) k_{2}^{p-2}\left(\mu-h_{2}\right) \in E[X]
$$

where $k_{2}=p^{-1}\left(\mu-h_{2}\right)^{p} \in A \backslash P$. Set $\mathscr{R} A:=E\left[\eta_{1}\right]$. From 2.2, $\mathscr{R} A$ is regular in codimension one if height one primes in $\mathscr{R} A$ lying over $P_{1} E$ are regular. From the above integral equation for $\eta_{1}$ over $E$, it is clear that the only such height one prime in $\mathscr{R} A$ is $Q_{1}:=$ $\left(p, \omega-h_{1}, \tau, \mu-h_{2}, \eta_{1}\right)$. Now $Q_{1_{Q_{1}}}=\left(\omega-h_{1}, \mu-h_{2}, \eta_{1}\right)_{Q_{1}}$. But $\eta_{1}\left(\mu-h_{2}\right)=k_{2}\left(\omega-h_{1}\right)$ and $k_{2} \notin Q_{1}$. Further, since $\tau-c_{1}^{\prime} \notin Q_{1}, Q_{1_{Q_{1}}}=\left(\eta_{1}\right)_{Q_{1}}$. Therefore $\mathscr{R} A$ is regular in codimension one.

(b) Set $Z:=<T>_{S}$ where $T=T_{1} \cup T_{2} \cup T_{3}$ and

$$
\begin{gathered}
T_{1}:=\left\{\left(\omega-h_{1}\right)^{i}\left(\mu-h_{2}\right)^{j} \mid i+j<p, 0 \leq i \leq p-2,0 \leq j \leq p-1\right\} \\
T_{2}:=\left\{p^{-1}\left(\omega-h_{1}\right)^{i}\left(\mu-h_{2}\right)^{j} \mid i+j \geq p, 1 \leq i \leq p-1,1 \leq j \leq p-1\right\} \\
T_{3}:=\left\{\tau-c_{1}^{\prime}=p^{-1}\left(\omega-h_{1}\right)^{p-1}\right\}
\end{gathered}
$$

For every $0 \leq i, j \leq p-1$, there is a unique element in $T$ with "leading coefficient" $\omega^{i} \mu^{j}$. Therefore the order of $T$ is $p^{2}$. Moreover since $A$ is $S$-free with a basis given by $D:=\left\{\left(\omega-h_{1}\right)^{i}\left(\mu-h_{2}\right)^{j} \mid 0 \leq i, j \leq p-1\right\}$, the elements of $T$ are linearly independent over $S$. From the relations

$$
\begin{gathered}
\left(\omega-h_{1}\right)^{p}=a^{\prime} p^{2}-p c_{1}^{\prime}\left(\omega-h_{1}\right) \\
\left(\mu-h_{2}\right)^{p}=b p-p c_{2}^{\prime}\left(\mu-h_{2}\right)
\end{gathered}
$$

we see that $Z$ is a ring. Since $Z$ satisfies $S_{2}$, if we show that $\mathscr{R} A \subseteq Z$, then from $2.2 Z=R$. It now suffices to note that $D \subseteq Z, \eta_{1} \in T$ and $\tau:=p^{-1}\left(\omega-h_{1}\right)^{p-1}+c_{1}^{\prime} \in Z$, so that $\mathscr{R} A \subseteq Z$. Thus $R=Z$ is $S$-free.

Lemma 3.2. With established notation, assume that $S[\omega]$ and $S[\mu]$ are integrally closed. The following hold

1. $\left(P^{(p-1)}\right)_{A}^{*}=\langle T\rangle_{S}$, where $P^{(p-1)}$ denotes the $(p-1)$-th symbolic power of the unique height one prime $P \subseteq A$ containing $p$ and $T:=T_{1} \cup T_{2}$, with

$$
\begin{gathered}
T_{1}:=\left\{\left(\omega-h_{1}\right)^{i}\left(\mu-h_{2}\right)^{j} \mid 0 \leq i, j \leq p-1, i+j<p\right\} \\
T_{2}:=\left\{p^{-1}\left(\omega-h_{1}\right)^{i}\left(\mu-h_{2}\right)^{j} \mid 0 \leq i, j \leq p-1, i+j \geq p\right\}
\end{gathered}
$$

2. The ring $A / P^{(p-1)}$ is Cohen-Macaulay. 
Proof. For every $0 \leq i, j \leq p-1$, there is a unique element in $T$ with "leading coefficient" $\omega^{i} \mu^{j}$. Therefore the order of $T$ is $p^{2}$. Moreover since $A$ is $S$-free with a basis given by $D:=\left\{\left(\omega-h_{1}\right)^{i}\left(\mu-h_{2}\right)^{j} \mid 0 \leq i, j \leq p-1\right\}$, the elements of $T$ are linearly independent over $S$. From the relations

$$
\begin{aligned}
& \left(\omega-h_{1}\right)^{p}=a p-p c_{1}^{\prime}\left(\omega-h_{1}\right) \\
& \left(\mu-h_{2}\right)^{p}=b p-p c_{2}^{\prime}\left(\mu-h_{2}\right)
\end{aligned}
$$

we see that $\langle T\rangle_{S}$ is a ring (in particular it is an $A$-module). Moreover, since it is CohenMacaulay, (2) immediately follows from (1) by using 2.4. Therefore, only (1) remains to be shown. Since $\left(P^{(p-1)}\right)^{*}$ and $\langle T\rangle_{S}$ are birational, $S_{2} A$-modules, it suffices to show their equality in codimension one. If $Q \subseteq A, Q \neq P$ is a height one prime, the equality is clear. So localize $A$ at $P$ and assume $(A, P)$ local for the rest of the proof. Then $\langle T\rangle_{S}=A[\eta]$ where $\eta:=\left(\omega-h_{1}\right)^{-1}\left(\mu-h_{2}\right)$. Note that $A=B^{\prime} /(G(U))$, where $B^{\prime}:=S[\omega][U]$. Set $\tilde{I}:=\left(\omega-h_{1}, U-h_{2}\right)^{p-1} \subseteq B^{\prime}$. We have $G(U) \in \tilde{I}$ :

$$
\begin{aligned}
U^{p}-g & =\left(U-h_{2}\right)^{p}+p\left(C_{2}^{\prime}\left(U-h_{2}\right)-b\right) \\
& =-k_{1}^{-1}\left(\omega-h_{1}\right)\left(C_{2}^{\prime}\left(U-h_{2}\right)-b\right) \cdot \Delta_{1}+0 \cdot \Delta_{2}+\cdots+0 \cdot \Delta_{p-1}-\left(U-h_{2}\right) \cdot \Delta_{p}
\end{aligned}
$$

where $\Delta_{i}=(-1)^{i} e_{i}$ with $e_{i}=\left(\omega-h_{1}\right)^{p-i}\left(U-h_{2}\right)^{i-1}$ and $k_{1}=p^{-1}\left(\omega-h_{1}\right)^{p}$. That is $\tilde{I}$ is the lift to $B^{\prime}$ of $P^{p-1}$. Further, $\tilde{I}$ is grade two perfect since it arises as the ideal of maximal minors of the $p \times(p-1)$ matrix $M$ :

$$
M=\left[\begin{array}{ccccc}
U-h_{2} & 0 & \cdots & 0 & 0 \\
\omega-h_{1} & U-h_{2} & 0 & \cdots & 0 \\
0 & \omega-h_{1} & U-h_{2} & \cdots & 0 \\
\vdots & 0 & \ddots & \ddots & \vdots \\
0 & \cdots & 0 & \omega-h_{1} & U-h_{2} \\
0 & \cdots & 0 & 0 & \omega-h_{1}
\end{array}\right]
$$

Let $M^{\prime}$ be the $p \times p$ matrix obtained by adjoining $M$ with the column of coefficients of $G(U)$ :

$$
M^{\prime}=\left[\begin{array}{cccccc}
U-h_{2} & 0 & \ldots & 0 & 0 & -k_{1}^{-1}\left(\omega-h_{1}\right)\left(C_{2}^{\prime}\left(U-h_{2}\right)-b\right) \\
\omega-h_{1} & U-h_{2} & 0 & \ldots & 0 & 0 \\
0 & \omega-h_{1} & U-h_{2} & \ldots & 0 & 0 \\
\vdots & 0 & \ddots & \ddots & \vdots & \vdots \\
0 & \ldots & 0 & \omega-h_{1} & U-h_{2} & 0 \\
0 & \ldots & 0 & 0 & \omega-h_{1} & -\left(U-h_{2}\right)
\end{array}\right]
$$

By [KU97][Lemma 2.5] (or Kat99][Prop 2.1]), $\left(P^{p-1}\right)^{*}$ is generated as an $A$-module by the set $\left\{\delta_{i}^{-1} M_{i, i}^{\prime} \mid 1 \leq i \leq p\right\}$, where $\delta_{i}$ denotes the image of $\Delta_{i}$ in $A$ and $M_{i, i}^{\prime}$ the image 
in $A$ of the $(i, i)$-th cofactor of $M^{\prime}$. This is exactly the set $\left\{\eta^{p-1}, \eta^{p-2}, \ldots, \eta, 1\right\}$. Since $\eta$ satisfies the integral equation $X^{p}-k_{1}^{-1} k_{2} \in A[X]\left(\right.$ with $\left.k_{2}=p^{-1}\left(\mu-h_{2}\right)^{p}\right)$, this implies $\left(P^{p-1}\right)^{*}=A[\eta]=\langle T\rangle_{S}$. Thus the proof is complete.

Proposition 3.3. With established notation, $R$ is $S$-free if $S[\omega]$ and $S[\mu]$ are integrally closed and $f g^{i} \notin S^{p \wedge p^{2}}$ for $1 \leq i \leq p-1$. Further in this case, $P^{(p-1)}$ is the conductor of $R$ to $A$ where $P$ is the unique height one prime in $A$ containing $p$ and $P^{(p-1)}$ denotes the $(p-1)$-th symbolic power of $P$.

Proof. Since $S[\omega]$ and $S[\mu]$ are integrally closed, we have from 2.11 that $f, g \notin S^{p \wedge p^{2}}$. Write $f=h_{1}^{p}+a p$ and $g=h_{2}^{p}+b p$ with $a, b \notin p S$. We first note the following: The condition $f g^{i} \notin S^{p \wedge p^{2}}$ for all $1 \leq i \leq p-1$ is equivalent to the condition $\prod_{i=1}^{p-1}\left(a h_{2}^{p}+i b h_{1}^{p}\right) \notin p S$. This follows since for $1 \leq i \leq p-1$

$$
\begin{aligned}
f g^{i} & =\left(h_{1}^{p}+a p\right)\left(h_{2}^{p}+b p\right)^{i} \\
& =\left(h_{1} h_{2}^{i}\right)^{p}+h_{2}^{p(i-1)}\left(a h_{2}^{p}+i b h_{1}^{p}\right) \cdot p+q \cdot p^{2}
\end{aligned}
$$

for some $q \in S$. We organize the proof as follows:

1. We first construct the normalization of $A$ locally at $N N L_{1}(A)$. Since $N N L_{1}(A)=$ $\{P\}$, we only need to construct $R_{P}$.

2. Using (1), identify a finite birational overring $A \hookrightarrow \mathscr{R} A$ such that $\mathscr{R} A$ satisfies $R_{1}$. Then choose a suitable finite birational overring $\mathscr{R} A \hookrightarrow Z$ such that $Z$ is $S$-free. This would show $R=Z$ is $S$-free.

1. From 2.3, $A$ is regular in codimension one outside of $V(p)$. Moreover $P:=(p, \omega-$ $\left.h_{1}, \mu-h_{2}\right)$ is the only height one prime in $A$ containing $p$. Localize at $P$ and assume $(A, P)$ local for part (1). Now $\left(\omega-h_{1}\right)^{p}=p k_{1}$ and $\left(\mu-h_{2}\right)^{p}=p k_{2}$ where $k_{1}=a-c_{1}^{\prime}\left(\omega-h_{1}\right)$ and $k_{2}=b-c_{2}^{\prime}\left(\mu-h_{2}\right)$. Since $k_{1}, k_{2} \notin P$, we have $P=\left(\omega-h_{1}, \mu-h_{2}\right)$. The element $\eta=\left(\omega-h_{1}\right)^{-1}\left(\mu-h_{2}\right) \in K$ satisfies the integral equation $l(X):=X^{p}-k_{1}^{-1} k_{2} \in A[X]$. If $\eta \in A$, then $P_{P}=\left(\omega-h_{1}\right)_{P}$ so $A$ is integrally closed. But from Sri21][Proposition $2.7]$ this is impossible. Therefore $A[\eta]$ is a proper birational extension of $A$. We claim that $E:=A[\eta]$ is regular when $\prod_{i=1}^{p-1}\left(a h_{2}^{p}+i b h_{1}^{p}\right) \notin p S$. We will observe that $E$ is local with maximal ideal $Q \subseteq E$ either of the form $Q=P E$ or $Q=(P, \eta-r) E$ for some suitable $r \in S \backslash p S$. To see this, let $\phi: \tilde{E}:=S[W, U]_{\tilde{P}}[X] \longrightarrow E$ be the natural projection map sending $W \mapsto \omega, U \mapsto \mu, X \mapsto \eta$, where $W, U, X$ are indeterminates over $S$ and $\tilde{P}:=\left(p, W-h_{1}, U-h_{2}\right)$. Let $Q \subseteq E$ be any maximal ideal and let $\tilde{Q}$ be the preimage of $Q$ under $\phi$. Now

$$
l(X) \equiv X^{p}-b a^{-1} \in(A / P)[X] \simeq(S / p S)[X]
$$

Since $S / p S$ is a field, if $l(X)$ is an irreducible polynomial over $(S / p S)[X]$ then

$$
\left(p, W-h_{1}, U-h_{2}, l(X)\right) \subseteq \tilde{Q}
$$


is a height four prime containing $p$. Therefore the above inclusion must be an equality. If on the other hand $l(X)$ is reducible over $S / p S$ then $l(X) \equiv(X-r)^{p} \in(S / p S)[X]$ for some $r \in S \backslash p S$. So in this case

$$
\left(p, W-h_{1}, U-h_{2}, X-r\right) \subset \tilde{Q}
$$

is a height four prime. Again, the above inclusion must then be an equality. Therefore in either case $E$ is local and the maximal ideal is either of the form $P E=\left(\omega-h_{1}, \mu-h_{2}\right) E$ or $Q:=\left(\omega-h_{1}, \mu-h_{2}, \eta-r\right) E$. In the first case, since $\eta \cdot\left(\omega-h_{1}\right)=\mu-h_{2}, E$ is a DVR. In the second case, we have $Q_{Q}=\left(\omega-h_{1}, \eta-r\right)_{Q}$. We now show that $Q_{Q}=(\eta-r)_{Q}$. We have for some $m \in E$ :

$$
\begin{aligned}
(\eta-r)^{p} & =\eta^{p}-b a^{-1}+p m \\
& =k_{1}^{-1} k_{2}-b a^{-1}+p m \\
& =k_{1}^{-1}\left[-c_{2}^{\prime}\left(\mu-h_{2}\right)+b a^{-1} c_{1}^{\prime}\left(\omega-h_{1}\right)+p m k_{1}\right] \\
& =k_{1}^{-1}\left(\omega-h_{1}\right)\left[-c_{2}^{\prime} \eta+b a^{-1} c_{1}^{\prime}+\left(\omega-h_{1}\right)^{p-1} m\right]
\end{aligned}
$$

So $Q$ is principal if $\alpha:=c_{2}^{\prime} \eta-b a^{-1} c_{1}^{\prime}$ is invertible in $E$. To show $\alpha \in E$ is a unit, it suffices to show $\left(a c_{2}^{\prime} \eta-b c_{1}^{\prime}\right)^{p} \in E$ is invertible. From $2.9, c_{1}^{\prime} \equiv h_{1}^{p-1} \bmod Q$ and $c_{2}^{\prime} \equiv h_{2}^{p-1} \bmod Q$. We then have

$$
\begin{aligned}
\left(a c_{2}^{\prime} \eta-b c_{1}^{\prime}\right)^{p} & \equiv a^{p}\left(c_{2}^{\prime}\right)^{p} \eta^{p}-b^{p}\left(c_{1}^{\prime}\right)^{p} \bmod Q \\
& \equiv b\left(a^{p-1}\left(c_{2}^{\prime}\right)^{p}-b^{p-1}\left(c_{1}^{\prime}\right)^{p}\right) \bmod Q \\
& \equiv b\left[a^{p-1}\left(h_{2}^{p-1}\right)^{p}-b^{p-1}\left(h_{1}^{p-1}\right)^{p}\right] \bmod Q \\
& \equiv b \prod_{i=1}^{p-1}\left(a h_{2}^{p}+i b h_{1}^{p}\right) \bmod Q
\end{aligned}
$$

Thus $\alpha \in E$ is a unit. Hence $E=A[\eta]$ is regular, that is $E=R$.

2. Set $\mathscr{R} A:=A\left[k_{1} \eta\right]$ for $\eta=\left(\omega-h_{1}\right)^{-1}\left(\mu-h_{2}\right)$ and $k_{1}=p^{-1}\left(\omega-h_{1}\right)^{p}$. Note that $k_{1} \eta=p^{-1}\left(\omega-h_{1}\right)^{p-1}\left(\mu-h_{2}\right)$ and that it satisfies the integral equation $X^{p}-k_{1}^{p-1} k_{2} \in A[X]$ for $k_{2}:=p^{-1}\left(\mu-h_{2}\right)^{p}$. Since $k_{1} \notin P$, by 2.2 and part (1) of the proof, $\mathscr{R} A$ is regular in codimension one.

Set $Z:=\langle T\rangle_{S}$ where $T$ is as in the statement of 3.2 . We see that $Z$ is a ring from the relations

$$
\begin{aligned}
& \left(\omega-h_{1}\right)^{p}=a p-p c_{1}^{\prime}\left(\omega-h_{1}\right) \\
& \left(\mu-h_{2}\right)^{p}=b p-p c_{2}^{\prime}\left(\mu-h_{2}\right)
\end{aligned}
$$

Moreover it is a free $S$-module of rank $p^{2}$. Clearly $\mathscr{R} A \subseteq Z$, so $Z$ inherits $R_{1}$ from $\mathscr{R} A$. Thus $Z=R$ and $R$ is $S$-free. 
Finally, from $3.2(1) P^{(p-1)}$ is contained in the conductor $J$ of $R$ to $A$. Since $A_{P}$ is a one dimensional Gorenstein local ring, $J_{P}=\left(P^{p-1}\right)_{P}$ and thus $J \subseteq P^{(p-1)}$. Thus $P^{(p-1)}$ is the conductor of $R$ to $A$.

Remark 3.4. The condition $f g^{i} \notin S^{p \wedge p^{2}}$ for $1 \leq i \leq p-1$ in 3.3 is saying that some suitable subrings of $A$ are integrally closed. As noted in the proof of 3.3 , the condition is equivalent to $\prod_{i=1}^{p-1}\left(a h_{2}^{p}+i b h_{1}^{p}\right) \notin p S$. Let $1 \leq k, i \leq p-1$ and $1 \leq i(k) \leq p-1$ be such that $i(k)-i k \in p \mathbb{Z}$. The condition $\prod_{i=1}^{p-1}\left(a h_{2}^{p}+i b h_{1}^{p}\right) \notin p S$ is saying that for all $1 \leq i \leq p-1$, $A_{i}:=S\left[\omega \mu^{i(1)}, \ldots, \omega^{j} \mu^{i(j)}, \ldots, \omega^{p-1} \mu^{i(p-1)}\right]$ is integrally closed. Indeed

$$
\left(\omega \mu^{i}\right)^{p}=f g^{i}=\left(h_{1} h_{2}^{i}\right)^{p}+\left(a h_{2}^{i p}+i b h_{1}^{p} h_{2}^{i p-p}\right) p+p^{2} q
$$

for some $q \in S$. If $i=1$, we have that $f g$ is squarefree in $S$ and by $2.11 S[\omega \mu]$ is integrally closed. If $i \neq 1$, the given condition is equivalent to saying that

$$
N N L_{1}\left(S\left[\omega \mu^{i}\right]\right) \cap V(p)=\emptyset
$$

Moreover $N N L_{1}\left(S\left[\omega \mu^{i}\right]\right) \subseteq V(g)$. Choose $k$ such that $i(k)=1$, so that $S\left[\omega \mu^{i}, \omega^{k} \mu\right]$ is a finite birational extension of both $S\left[\omega \mu^{i}\right]$ and $S\left[\omega^{k} \mu\right]$. Now $N N L_{1}\left(S\left[\omega^{k} \mu\right]\right) \subseteq V(f)$. So by 2.2. $S\left[\omega \mu^{i}, \omega^{k} \mu\right]$ is regular in codimension one since $f, g \in S$ satisfy $\mathscr{A}_{1}$. Since $A_{i}$ is a finite birational extension of $S\left[\omega \mu^{i}, \omega^{k} \mu\right]$, it is regular in codimension one for the same reason. The remark follows since it is easily checked that $A_{i}$ is $S$-free.

Remark 3.5. The powers of the prime $P^{p-1} \subseteq A$ in 3.3 are not $P$-primary in general. For example if $p=3$, observe that $3 a, 3 b \in P^{2}$. However, it holds that $P^{(p-1)}=(p)+P^{p-1}$.

\section{Existence of birational MCM modules}

In this section we look at cases where $R$ is not $S$-free. That is in the primary case of interest, when $S$ is an unramified regular local ring of mixed characteristic $p$, we look at non-CohenMacaulay integral closures $R$. More specifically, we will show that $p . d \cdot S(R)=1$ under some natural conditions and show that in this case $R$ admits a birational MCM module.

From 3.1 and 3.3 , if we are looking for a non $S$-free $R$, we must have that $S[\omega]$ and $S[\mu]$ are integrally closed such that there exists an $1 \leq i \leq p-1$ satisfying $f g^{i} \in S^{p \wedge p^{2}}$. The reader can easily see that if it exists, such an " $i$ " is unique. We start by identifying an ideal $I \subseteq A$, such that $I^{*}=R$ under this circumstance.

Convention 4.1. We maintain notation as set up in 2.6 and make the additional assumption that $f, g \notin S^{p \wedge p^{2}}$. Write $f=h_{1}^{p}+a p, g=h_{2}^{p}+b p$ with $a, b \notin p S$. Assume further that $h_{1}, h_{2} \neq 0$. Note here that if $h_{1}=0$ (or $h_{2}=0$ ), we have by 3.3 that $R$ is $S$-free. Let $P:=\left(p, \omega-h_{1}, \mu-h_{2}\right) \subseteq A$ denote the unique height one prime in $A$ containing $p$. 
Lemma 4.2. For $H:=\left(p, \omega \mu^{i}-h_{1} h_{2}^{i}\right) \subseteq A, H_{P}^{*}=\left\langle 1, \tau_{i}\right\rangle_{A_{P}}$ where

$$
\tau_{i}=p^{-1}\left[\left(\omega \mu^{i}\right)^{p-1}+h_{1} h_{2}^{i}\left(\omega \mu^{i}\right)^{p-2}+\cdots+\left(h_{1} h_{2}^{i}\right)^{p-1}\right] \in K
$$

Proof. Localize $A$ at $P$ and assume $(A, P)$ local. Consider the ideal

$$
\tilde{H}:=\left(p, W \mu^{i}-h_{1} h_{2}^{i}\right) \subseteq S[\mu][W]
$$

We have $F(W) \in \tilde{H}$ :

$$
F(W)-h_{2}^{-i p}\left[\left(W \mu^{i}\right)^{p-1}+h_{1} h_{2}^{i}\left(W \mu^{i}\right)^{p-2}+\cdots+\left(h_{1} h_{2}^{i}\right)^{p-1}\right] \cdot\left(W \mu^{i}-h_{1} h_{2}^{i}\right) \in p S[\mu][W]
$$

Clearly $\tilde{H}$ is a grade two perfect ideal in $S[\mu][W]$ and is the ideal of maximal minors of the matrix $E$ :

$$
E=\left[\begin{array}{c}
W \mu^{i}-h_{1} h_{2}^{i} \\
p
\end{array}\right]
$$

Adjoining the column of coefficients from (44) appropriately, we have for some $\alpha \in S[\mu[[W]$ the matrix $E^{\prime}$ :

$$
E^{\prime}=\left[\begin{array}{cc}
W \mu^{i}-h_{1} h_{2}^{i} & \alpha \\
p & h_{2}^{-i p}\left[\left(W \mu^{i}\right)^{p-1}+h_{1} h_{2}^{i}\left(W \mu^{i}\right)^{p-2}+\cdots+\left(h_{1} h_{2}^{i}\right)^{p-1}\right]
\end{array}\right]
$$

From [Kat99] [Proposition 2.1], $H^{*}=\left\langle E_{11}^{\prime} / \delta_{1}, E_{22}^{\prime} / \delta_{2}\right\rangle_{A}$ where $E_{i i}^{\prime}$ and $\delta_{i}$ denote the image in $A$ of the $(i, i)$-th cofactor of $E^{\prime}$ and the $i$-th (signed) minor of $E$ respectively. Thus $H^{*}=\left\langle 1, \tau_{i}\right\rangle_{A}$

Lemma 4.3. With established notation, let $f g^{i} \in S^{p \wedge p^{2}}$. Then for

$$
I:=p A+P^{p-2} \cdot\left(\omega \mu^{i}-h_{1} h_{2}^{i}\right) A
$$

we have $I_{A}^{*}=R$.

Proof. Since $I_{A}^{*}$ and $R$ are birational $S_{2} A$-modules, it suffices to show the desired equality in codimension one. If $Q \neq P$ is a height one prime in $A, I_{Q}^{*}=R_{Q}=A_{Q}$. Therefore localize $A$ at $P$ and assume $(A, P)$ and $(S, p S)$ are one dimensional local rings for the remainder of the proof.

We have $A=S\left[\mu, \omega \mu^{i}\right]$. Note that $g, f g^{i} \in S$ are units and therefore trivially are square free and satisfy $\mathscr{A}_{1}$. Moreover, $S[\mu]$ is integrally closed and $S\left[\omega \mu^{i}\right]$ is not. Thus we are in the setting of $3.1(2)$. From the proof of 3.1 (2)(a), we get that $R=A[\tau, \eta]$ where $\tau=p^{-1}\left[\left(\omega \mu^{i}\right)^{p-1}+h_{1} h_{2}^{i}\left(\omega \mu^{i}\right)^{p-2}+\cdots+\left(h_{1} h_{2}^{i}\right)^{p-1}\right]$ and $\eta=p^{-1}\left(\omega \mu^{i}-h_{1} h_{2}^{i}\right)\left(\mu-h_{2}\right)^{p-1}$. Since $\tau \eta \in A$, we see from 2.11(c) and equation (2) that $R=\left\langle 1, \eta, \ldots, \eta^{p-2}, \tau\right\rangle_{A}$.

Since $p \in P^{p-1}$, a straightforward calculation gives

$$
P^{p-1} \cap\left(p, \omega \mu^{i}-h_{1} h_{2}^{i}\right)=(p)+P^{p-1} \cap\left(\omega \mu^{i}-h_{1} h_{2}^{i}\right)=(p)+P^{p-2} \cdot\left(\omega \mu^{i}-h_{1} h_{2}^{i}\right)=I
$$


From 3.2, $\left(P^{p-1}\right)^{*}=A[\eta] \subseteq R$. Since $A$ is Gorenstein, $A:_{K} R \subseteq P^{p-1}$. Let $H$ be as in 4.2. Combining 4.2 and 2.11(c), we get that $H^{*}=A[\tau] \subseteq R$. Again since $H$ is reflexive, $A:_{K} R \subseteq H$. Therefore, $A:_{K} R \subseteq P^{p-1} \cap H=I$.

To show $I R \subseteq A$, note that $I \eta^{i} \subseteq A$ for $1 \leq i \leq p-2$, since $I \subseteq P^{p-1}=A[\eta]^{*}$. Similarly, $I \tau \subseteq A$ since $I \subseteq H=A[\tau]^{*}$. Thus we have shown $I=A:_{K} R$ and the proof is complete.

We now set out to show $R$ need not be Cohen-Macaulay.

Lemma 4.4. Let $S[\omega], S[\mu]$ be integrally closed such that $f g \in S^{p \wedge p^{2}}$. Then

1. $R \subseteq\left\langle\{1\} \cup p^{-1} \cdot\left(\omega-h_{1}, \mu-h_{2}\right)^{p-1}\right\rangle_{A}$.

2. Consider $y=p^{-1}\left(\sum_{i=1}^{p} a_{i}\left(\mu-h_{2}\right)^{p-i}\left(\omega-h_{1}\right)^{i-1}\right) \in K$ with the $a_{i} \in A$. Then $y \in R$ if and only if for all $2 \leq i \leq p, a_{i-1} h_{2}+a_{i} h_{1} \in P$.

Proof. From 4.3, $p \cdot R \subseteq A$, so consider an arbitrary element $y:=p^{-1} \cdot x \in R$ with $x \in A$. From 4.3, $x \cdot\left(\omega-h_{1}\right)^{p-2}\left(\omega \mu-h_{1} h_{2}\right) \in p A$. Lifting to $B:=S[W, U]$ and denoting lifts by

$$
\tilde{x}\left(W-h_{1}\right)^{p-2}\left(W U-h_{1} h_{2}\right) \in(p, F(W), G(U))
$$

Write

$$
\omega \mu-h_{1} h_{2}=\left(\omega-h_{1}\right)\left(\mu-h_{2}\right)+h_{2}\left(\omega-h_{1}\right)+h_{1}\left(\mu-h_{2}\right)
$$

Lifting the identity in (6) (6) $B$ we see that $\tilde{x} \in\left(p, W-h_{1},\left(U-h_{2}\right)^{p-1}\right)$. By symmetry $\tilde{x} \in\left(p,\left(W-h_{1}\right)^{p-1}, U-h_{2}\right)$ and hence

$$
\tilde{x} \in\left(p,\left(U-h_{2}\right)^{p-1},\left(W-h_{1}\right)^{p-1},\left(W-h_{1}\right)\left(U-h_{2}\right)\right)
$$

This is because for a regular sequence $(q, y, z) \subseteq B$

$$
\left(q, y, z^{n}\right) \cap\left(q, y^{n}, z\right)=\left(q, y^{n}, z^{n}, y z\right)
$$

Since $1 \in R$, towards describing $A$-module generators for $R$ we may assume that $y=p^{-1} x$ with

$$
x=a_{1} \cdot\left(\mu-h_{2}\right)^{p-1}+a_{2} \cdot\left(\omega-h_{1}\right)^{p-1}+a_{3} \cdot\left(\omega-h_{1}\right)\left(\mu-h_{2}\right)
$$

for some $a_{1}, a_{2}, a_{3} \in A$. Suppose we can write

$$
y=p^{-1}\left[a_{1}\left(\mu-h_{2}\right)^{p-1}+a_{2}\left(\omega-h_{1}\right)\left(\mu-h_{2}\right)^{p-2}+\cdots+a_{p}\left(\omega-h_{1}\right)^{p-1}+b \cdot\left(\omega-h_{1}\right)^{i}\left(\mu-h_{2}\right)^{i}\right]
$$

with $1 \leq i<(p-1) / 2$ and $a_{i}, b \in A$. By (8), we can do this for $i=1$, where $a_{j}=0$ for $2 \leq j \leq p-1$. Now using (6) we get that $y \cdot\left(\omega-h_{1}\right)^{p-1-i}\left(\mu-h_{2}\right)^{i-1}\left(\omega \mu-h_{1} h_{2}\right) \in p A$ if and only if

$a_{i+1} h_{1}\left(\omega-h_{1}\right)^{p-1}\left(\mu-h_{2}\right)^{p-1}+a_{i} h_{2}\left(\omega-h_{1}\right)^{p-1}\left(\mu-h_{2}\right)^{p-1}+b h_{1}\left(\omega-h_{1}\right)^{p-1}\left(\mu-h_{2}\right)^{2 i} \in p A$ 
Pulling back to $B$

$$
\tilde{b} h_{1}\left(U-h_{2}\right)^{2 i}+h_{2} \tilde{a}_{i}\left(U-h_{2}\right)^{p-1}+h_{1} \tilde{a}_{i+1}\left(U-h_{2}\right)^{p-1} \in\left(p,\left(U-h_{2}\right)^{p}, W-h_{1}\right)
$$

and thus $\tilde{b} \in\left(p, W-h_{1},\left(U-h_{2}\right)^{p-2 i-1}\right)$. By symmetry, $\tilde{b} \in\left(p, U-h_{2},\left(W-h_{1}\right)^{p-2 i-1}\right)$ and hence by (7)

$$
\tilde{b} \in\left(p,\left(U-h_{2}\right)^{p-2 i-1},\left(W-h_{1}\right)^{p-2 i-1},\left(W-h_{1}\right)\left(U-h_{2}\right)\right)
$$

Therefore

$$
p y \in\left(p,\left(\omega-h_{1}\right)^{i+1}\left(\mu-h_{2}\right)^{i+1}\right)+\left(\omega-h_{1}, \mu-h_{2}\right)^{p-1}
$$

Starting from (8) and iterating the argument from (9) to this point sufficiently many times, we see that

$$
R \subseteq\left\langle\{1\} \cup p^{-1} \cdot\left(\omega-h_{1}, \mu-h_{2}\right)^{p-1}\right\rangle_{A}
$$

Consider

$$
y=p^{-1}\left(\sum_{i=1}^{p} a_{i}\left(\mu-h_{2}\right)^{p-i}\left(\omega-h_{1}\right)^{i-1}\right) \in K
$$

with the $a_{i} \in A$. From 4.3, $y \in R$ if and only if for all $2 \leq i \leq p$

$$
y \cdot\left(\omega-h_{1}\right)^{p-i}\left(\mu-h_{2}\right)^{i-2}\left(\omega \mu-h_{1} h_{2}\right) \in A
$$

From (6) the above statements are equivalent to

$$
\left(a_{i-1} h_{2}+a_{i} h_{1}\right)\left(\mu-h_{2}\right)^{p-1}\left(\omega-h_{1}\right)^{p-1} \in p A
$$

for each $2 \leq i \leq p$. Lifting to $B$, we see that (13) is equivalent to

$$
a_{i-1} h_{2}+a_{i} h_{1} \in P
$$

Thus the proof is complete.

Lemma 4.5. Assume $S$ is an unramified regular local ring of mixed characteristic $p \geq 3$. Let $S[\omega], S[\mu]$ be integrally closed such that $f g \in S^{p \wedge p^{2}}$. Then $\nu_{S}(R) \leq p^{2}+1$. More explicitly, set $\eta_{i}:=p^{-1}\left(\omega-h_{1}\right)^{i}\left(\mu-h_{2}\right)^{p-i}$ for $1 \leq i \leq p-1$. We have $R=\left\langle A\left[\eta_{1}, \ldots, \eta_{p-1}\right] \cup\{\epsilon\}\right\rangle_{S}$ for:

$$
\epsilon:=p^{-1} \sum_{i=1}^{p}(-1)^{i} c^{p-i} e^{i-1}\left(\mu-h_{2}\right)^{p-i}\left(\omega-h_{1}\right)^{i-1}
$$

where $h_{1} \equiv z c \bmod p S, h_{2} \equiv z e \bmod p S$ for some $z \in S \backslash p S$ and $c, e \in S$ relatively prime. 
Proof. Suppose $\langle T\rangle_{S}$ is as in 3.2 . Note that it is just the ring $A\left[\eta_{1}, \ldots, \eta_{p-1}\right]$. Since $\langle T\rangle_{S}$ is $S$-free of rank $p^{2}$, the assertion $\nu_{S}(R) \leq p^{2}+1$ follows from the second assertion.

Since $S / p S$ is regular local $\left(\right.$ a UFD), $h_{1} \equiv(z c) \bmod p S, h_{2} \equiv(z e) \bmod p S$ for some $z \in S \backslash p S$ and $c, e \in S$ relatively prime. First suppose $c$ or $e$ is a unit in $S$. Then it follows from 4.4(1) and (2) that $R=\left\langle A\left[\eta_{1}, \ldots, \eta_{p-1}\right] \cup \epsilon\right\rangle_{A}$. Notice that if $i+j>0$, then $\left(\omega-h_{1}\right)^{i}\left(\mu-h_{2}\right)^{j} \in\left(A\left[\eta_{1}, \ldots, \eta_{p-1}\right]:_{K} \epsilon\right)$. Thus $R=\left\langle A\left[\eta_{1}, \ldots, \eta_{p-1}\right] \cup\{\epsilon\}\right\rangle_{S}$ in this case.

Next assume that neither $c$ or $e$ is a unit, so that $(p, c, e) \subseteq S$ forms a regular sequence. Now $\epsilon \in R$ from 4.4(2). From 4.4(1), it suffices to look at elements of the form

$$
y=p^{-1}\left(\sum_{i=1}^{p} a_{i}\left(\mu-h_{2}\right)^{p-i}\left(\omega-h_{1}\right)^{i-1}\right) \in R
$$

In view of $4.4(2)$, the condition $a_{1} h_{2}+a_{2} h_{1} \in P$ upon lifting to $B:=S[W, U]$ (denoting lifts by $\sim$ ) tells us that $\tilde{a_{1}}, \tilde{a_{2}}$ arise from the first syzygy of the grade five complete intersection $B$-ideal, $\tilde{Q}:=\left(p, c, e, W-h_{1}, U-h_{2}\right)$. In particular

$$
\tilde{a_{2}} \in\left(p, c, W-h_{1}, U-h_{2}\right) \cap\left(p, e, W-h_{1}, U-h_{2}\right)=\left(p, c e, W-h_{1}, U-h_{2}\right)
$$

since $a_{2} h_{2}+a_{3} h_{1} \in P$ as well. Since $A\left[\eta_{1}, \ldots, \eta_{p-1}\right] \subseteq R$, towards describing $A$-module generators for $R$ we may assume $\tilde{a_{2}}=\alpha c e$ for some $\alpha \in B$ and consequently that $\tilde{a_{1}}=-\alpha c^{2}$ and $\tilde{a_{3}}=-\alpha e^{2}$.

Now let $3 \leq i<p$ be such that for all $1 \leq k \leq i, a_{k}=(-1)^{k} \alpha c^{i-k} e^{k-1}$ for some $\alpha \in A$. Lifting $a_{i} h_{2}+a_{i+1} h_{1} \in P$ to $B$, we have $(-1)^{i} \alpha e^{i}+a_{i+1} c \in \tilde{P}$. Since $A\left[\eta_{1}, \ldots, \eta_{p-1}\right] \subseteq R$, we may assume that $\alpha=\alpha^{\prime} c$ for some $\alpha^{\prime} \in B$ and hence that $a_{i+1}=(-1)^{i+1} \alpha^{\prime} \cdot e^{i}$. Iterating this argument, we get that $R=\left\langle A\left[\eta_{1}, \ldots, \eta_{p-1}\right] \cup\{\epsilon\}\right\rangle_{A}$. Finally, if $i+j>0$ then $\left(\omega-h_{1}\right)^{i}\left(\mu-h_{2}\right)^{j} \in\left(A\left[\eta_{1}, \ldots, \eta_{p-1}\right]:_{K} \epsilon\right)$ and the conclusion follows.

Proposition 4.6. Let $(S, \mathfrak{m})$ be an unramified regular local ring of mixed characteristic $p \geq 3$ such that $S[\omega]$ and $S[\mu]$ are integrally closed and $f g \in S^{p \wedge p^{2}}$. Then $R$ is CohenMacaulay if and only if $Q:=\left(p, h_{1}, h_{2}\right) \subset S$ is a two generated ideal or all of $S$. Moreover, $p . d_{S}(R) \leq 1$.

Proof. Since $S / p S$ is a UFD, $h_{1} \equiv z c \bmod p S, h_{2} \equiv z e \bmod p S$ for some $z \in S \backslash p S$ and $c, e \in S$ relatively prime. Then $Q$ is a two generated ideal or all of $R$ if and only if $c$ or $e$ is a unit. From 4.5, $R=\left\langle A\left[\eta_{1}, \ldots, \eta_{p-1}\right] \cup\{\epsilon\}\right\rangle_{S}$. Suppose that $c$ is a unit. Then

$$
\left(\mu-h_{2}\right)^{p-1} \in\left\langle\epsilon,\left(\omega-h_{1}\right)\left(\mu-h_{2}\right)^{p-2}, \ldots,\left(\omega-h_{1}\right)^{p-1}\right\rangle_{S} .
$$

Thus $R$ is $S$-free of rank $p^{2}$ and hence is Cohen-Macaulay.

Now assume neither $c$ nor $e$ is a unit, that is $Q$ is either grade three perfect or grade two and not perfect. We know from 4.5 that $R=\left\langle A\left[\eta_{1}, \ldots, \eta_{p-1}\right] \cup\{\epsilon\}\right\rangle_{S}$. With $T$ as in 3.2, define $\Gamma: T \rightarrow \mathbb{Z}, \Gamma^{\prime}: T \rightarrow \mathbb{Z}$ by

$$
\Gamma\left(p^{-k}\left(\omega-h_{1}\right)^{i}\left(\mu-h_{2}\right)^{j}\right)=i+j
$$




$$
\Gamma^{\prime}\left(p^{-k}\left(\omega-h_{1}\right)^{i}\left(\mu-h_{2}\right)^{j}\right)=i
$$

Define a total ordering on $T$ as follows: for $x, y \in T$, if $\Gamma(x) \geq \Gamma(y)$ then $x \geq y$ and if $\Gamma(x)=$ $\Gamma(y)$, then $x \geq y$ if $\Gamma^{\prime}(x) \geq \Gamma^{\prime}(y)$. Let $\alpha: S^{p^{2}+1} \rightarrow R$ be the $S$-projection map defined by the generating set $T \cup\{\epsilon\}$ such that the basis element $e_{p^{2}+1}$ maps to $\epsilon$ and the image of the basis elements $e_{i}, i \neq p^{2}+1$ is defined by the ordered set $T$. Consider $U=\left[u_{i}\right] \in \operatorname{Ker}(\alpha)$. Since $A$ is $S$-free with a basis given by $\left\{\left(\omega-h_{1}\right)^{i}\left(\mu-h_{2}\right)^{j} \mid 0 \leq i, j \leq p-1\right\}$, we get that that $u_{i}=0$ for $m \leq i \leq p^{2}$, where $m=p^{2}-2^{-1}(p-1) p+1$. Let $p \epsilon=\sum_{i=1}^{m-1} v_{i} x_{i}$ with the $v_{i} \in S$ and $x_{i}$ from the ordered set $T$. Since $p \in S$ is prime we get the following free resolution of $R$ over $S$ :

$$
0 \longrightarrow S \stackrel{\psi^{T}}{\longrightarrow} S^{p^{2}+1} \stackrel{\alpha}{\longrightarrow} R \longrightarrow 0
$$

where $\psi=\left[v_{1} \ldots v_{m-1} 0 \ldots 0-p\right]$. The above resolution is minimal since $\psi^{T}(S) \subseteq \mathfrak{m} S^{p^{2}+1}$, so that $p \cdot d_{S}(R)=1$. The proof is now complete.

Remark 4.7. Let $S$ be an unramified regular local ring of mixed characteristic $p \geq 3$. Note that the ideal $\left(p, h_{1}, h_{2}\right) \subseteq S$ is a two generated ideal or all of $R$ if and only if the same property holds for the ideal $(p, f, g) \subseteq S$. Similarly, the ideal $\left(p, h_{1}, h_{2}\right)$ has grade three if and only if the ideal $(p, f, g)$ has the same property.

Example 4.8. The conditions in 4.6 give a non-empty class of non Cohen-Macaulay integral closures $R$. For an example where $Q=\left(p, h_{1}, h_{2}\right)$ has grade three, consider $S=\mathbb{Z}[X, Y]_{(3, X, Y)}$ where $X, Y$ are indeterminates over $\mathbb{Z}_{(3)}$. Let

$$
\begin{gathered}
f=-5 X^{3}+9=X^{3}+3\left(3-2 X^{3}\right) \\
g=-2 Y^{3}+9=Y^{3}+3\left(3-Y^{3}\right)
\end{gathered}
$$

and $\omega^{3}=f, \mu^{3}=g$. Then $f, g$ are square free elements that form a regular sequence in $S$. It is easily checked that $[K: L]=9$ and that this choice satisfies the hypothesis of [4.6, so that $p . d \cdot S(R)=1$.

For an example where $Q$ has grade two but $p \cdot d_{S}(S / Q)=3$, let $S=\mathbb{Z}[X, Y]_{(p, X, Y)}$ for some prime number $p \geq 3$. Set

$$
\begin{gathered}
f=(1-p) X^{2 p}+p^{2}=\left(X^{2}\right)^{p}+p\left(p-X^{2 p}\right) \\
g=(1+p)(X Y)^{p}+p^{2}=(X Y)^{p}+p\left(p+(X Y)^{p}\right)
\end{gathered}
$$

Then $f, g \in S$ are square free and form a regular sequence in $S$. It is easily verified that $[K: L]=p^{2}$ and that the choice satisfies the hypothesis of [4.6, so that p.d.S $(R)=1$.

Lemma 4.9. With established notation, the following holds

$$
P_{A}^{*}=\left\langle 1, p^{-1}\left(\omega-h_{1}\right)^{p-1}\left(\mu-h_{2}\right)^{p-1}\right\rangle_{A}
$$


Proof. Set $P_{1}:=\left(p, \omega-h_{1}\right)$ and $P_{2}:=\left(p, \mu-h_{2}\right)$, so that $P^{*}=P_{1}^{*} \cap P_{2}^{*}$. Let $\tilde{P}_{1}:=$ $\left(p, W-h_{1}\right) \subseteq S[\mu][W]$. It is the maximal minors of

$$
E=\left[\begin{array}{c}
W-h_{1} \\
p
\end{array}\right]
$$

We have $F(W) \in \tilde{P}_{1}, F(W)=a \cdot(-p)+\left(W^{p-1}+h_{1} W^{p-2}+\cdots+h_{1}^{p-1}\right)\left(W-h_{1}\right)$. Adjoining the appropriate column of coefficients

$$
E^{\prime}=\left[\begin{array}{cc}
W-h_{1} & a \\
p & W^{p-1}+\cdots+h_{1}^{p-1}
\end{array}\right]
$$

From [KU97] [Lemma 2.5] $P_{1}^{*}=\left\langle E_{11}^{\prime} / \delta_{1}, E_{22}^{\prime} / \delta_{2}\right\rangle_{A}$ where $E_{i i}^{\prime}$ and $\delta_{i}$ denote the image in $A$ of the $(i, i)$-th cofactor of $E^{\prime}$ and the $i$-th (signed) minor of $E$. Therefore

$$
P_{1}^{*}=\left\langle 1, p^{-1}\left(\omega^{p-1}+\cdots+h_{1}^{p-1}\right)\right\rangle_{A}
$$

Identically

$$
P_{2}^{*}=\left\langle 1, p^{-1}\left(\mu^{p-1}+\cdots+h_{2}^{p-1}\right)\right\rangle_{A}
$$

Now consider $y \in P^{*}=P_{1}^{*} \cap P_{2}^{*}$. Write for some $\alpha_{1}, \alpha_{2}, \beta_{1}, \beta_{2} \in A$

$$
p y=p \alpha_{1}+\beta_{1}\left(\omega^{p-1}+\cdots+h_{1}^{p-1}\right)=p \alpha_{2}+\beta_{2}\left(\mu^{p-1}+\cdots+h_{2}^{p-1}\right)
$$

Lifting to $B:=S[W, U]$ and denoting lifts by $\sim$

$$
p\left(\tilde{\alpha_{1}}-\tilde{\alpha_{2}}\right)+\tilde{\beta_{1}}\left(W^{p-1}+\cdots+h_{1}^{p-1}\right)-\tilde{\beta}_{2}\left(U^{p-1}+\cdots+h_{2}^{p-1}\right) \in(F(W), G(U))
$$

Writing $W^{p-1}+\cdots+h_{1}^{p-1}=\left(W-h_{1}\right)^{p-1}+p \cdot C_{1}^{\prime}\left(\right.$ respectively for $\left.U^{p-1}+\cdots+h_{2}^{p-1}\right)$,

$$
\tilde{\beta}_{1}\left(W-h_{1}\right)^{p-1}-\tilde{\beta}_{2}\left(U-h_{2}\right)^{p-1} \in(p, F(W), G(U))
$$

This gives $\tilde{\beta}_{1} \in\left(p, W-h_{1},\left(U-h_{2}\right)^{p-1}\right)$. Since $1 \in P^{*}$ and $\left(\omega-h_{1}\right)\left(\omega^{p-1}+\cdots+h_{1}^{p-1}\right) \in p A$, we get $P^{*} \subseteq\left\langle 1, p^{-1}\left(\omega-h_{1}\right)^{p-1}\left(\mu-h_{2}\right)^{p-1}\right\rangle_{A}$. Since the reverse inclusion is obvious, the proof is complete.

Theorem 4.10. Let $(S, \mathfrak{m})$ be an unramified regular local ring of mixed characteristic $p \geq 3$. Then

1. $R$ is Cohen-Macaulay if

(a) At least one of $S[\omega], S[\mu]$ is not integrally closed.

(b) $S[\omega], S[\mu]$ are integrally closed and $f g^{i} \notin S^{p \wedge p^{2}}$ for all $1 \leq i \leq p-1$. 
2. Let $S[\omega], S[\mu]$ be integrally closed and $f g \in S^{p \wedge p^{2}}$. Then $R$ is Cohen-Macaulay if and only if $Q:=(p, f, g) \subseteq S$ is a two generated ideal or all of $S$. Moreover, $p . d_{S}(R) \leq 1$ and $\nu_{S}(R) \leq p^{2}+1$.

3. If $Q:=(p, f, g) \subseteq S$ has grade three, $R$ admits a birational maximal Cohen-Macaulay module.

Proof. We have shown 1 (a) in 3.1 and 1 (b) in 3.3. The proof of (2) follows from 4.6, 4.5 and 4.7 .

Now assume $Q$ has grade three. From part (1), we may assume that $S[\omega], S[\mu]$ are integrally closed and $f g^{i} \in S^{p \wedge p^{2}}$ for some (unique) $1 \leq i \leq p-1$. From 4.3, $I^{*}=R$ for $I:=p A+\left(\omega \mu^{i}-h_{1} h_{2}^{i}\right) \cdot P^{p-2}$. Set $M:=(I P)^{*}$. Then $M$ is an $R$-module since $\left(A:_{K} I P\right)=\left(\left(A:_{K} I\right):_{K} P\right)=\left(R:_{K} P\right)$. We will show $\operatorname{depth}_{S}(M)=d$, so that $M$ is an MCM module over $R$. By definition

$$
M=(I P)^{*}=\left(p \cdot P+\left(\omega \mu^{i}-h_{1} h_{2}^{i}\right) \cdot P^{p-1}\right)^{*}=F_{1} \cap F_{2} .
$$

where $F_{1}=p^{-1} P^{*}$ and $F_{2}=\left(\omega \mu^{i}-h_{1} h_{2}^{i}\right)^{-1}\left(P^{p-1}\right)^{*}$. This is because for ideals $H, N \subseteq A$, $\left(A:_{K} H+N\right)=\left(A:_{K} H\right) \cap\left(A:_{K} N\right)$ as $A$-modules. Now $A / P \simeq S / p S$ as $S$-modules, therefore by the depth lemma $P$ is $S$-free. By 2.5. $\operatorname{Hom}_{A}(P, A) \simeq \operatorname{Hom}_{S}(P, S)$ as $S$ modules and hence $P^{*}$ is Cohen-Macaulay. On the other hand, since $\left(P^{p-1}\right)^{*}$ and $\left(P^{(p-1)}\right)^{*}$ are birational $S_{2}$ modules that agree in codimension one, we have $\left(P^{p-1}\right)^{*}=\left(P^{(p-1)}\right)^{*}$. From $3.2(2)$ and 2.4 we then have that $\left(P^{p-1}\right)^{*}$ is Cohen-Macaulay. Therefore $F_{1}$ and $F_{2}$ are Cohen-Macaulay since $F_{1} \simeq P^{*}$ and $F_{2} \simeq\left(P^{p-1}\right)^{*}$ as $A$-modules and $S$-modules. We have the natural short exact sequence of $S$-modules

$$
0 \longrightarrow F_{1} \cap F_{2} \longrightarrow F_{1} \oplus F_{2} \longrightarrow F_{1}+F_{2} \longrightarrow 0
$$

To complete the proof it suffices to show that $\operatorname{depth}_{S}\left(F_{1}+F_{2}\right) \geq d-1$. Set

$$
\mathscr{F}:=p\left(\omega \mu^{i}-h_{1} h_{2}^{i}\right) \cdot\left(F_{1}+F_{2}\right)=\mathscr{F}_{1}+\mathscr{F}_{2} .
$$

where $\mathscr{F}_{1}:=\left(\omega \mu^{i}-h_{1} h_{2}^{i}\right) P^{*}$ and $\mathscr{F}_{2}:=p\left(P^{p-1}\right)^{*}$. Clearly $F_{1}+F_{2} \simeq \mathscr{F}$ as $A$-modules and hence as $S$-modules. From $3.2(1), \mathscr{F}_{2}=(p)+P^{p}$ and from 4.9

$$
\mathscr{F}_{1}=\left(\omega \mu^{i}-h_{1} h_{2}^{i}, p^{-1}\left(\omega \mu^{i}-h_{1} h_{2}^{i}\right)\left(\omega-h_{1}\right)^{p-1}\left(\mu-h_{2}\right)^{p-1}\right) A
$$

Set $m:=\omega \mu^{i}-h_{1} h_{2}^{i}$. We make the following two claims:

1. $\mathscr{F}=\mathscr{F}_{2}+(m)$.

2. $\left(\mathscr{F}_{2}:_{A} m\right)=(p)+P^{p-1}$. 
Assume both claims hold. Since $\left(\mathscr{F}_{2}:_{A} m\right) \simeq \mathscr{F}_{2} \cap(m)$ as $A$-modules and hence $S$-modules, we have a natural short exact sequence of $S$-modules

$$
0 \longrightarrow(p)+P^{p-1} \longrightarrow \mathscr{F}_{2} \oplus(m) \longrightarrow 0
$$

If $\operatorname{depth}_{S}\left((p)+P^{p-1}\right)=d$, then $\operatorname{depth}_{S}(\mathscr{F}) \geq d-1$ and we are done. But $\operatorname{depth}_{S}((p)+$ $\left.P^{p-1}\right)=d$ if and only if $A /\left((p)+P^{p-1}\right)$ is Cohen-Macaulay. For $B:=S[W, U]_{\left(\mathfrak{m}, W-h_{1}, U-h_{2}\right)}$ we have as $B$-modules

$$
A /\left((p)+P^{p-1}\right) \simeq B /\left((p)+\left(W-h_{1}, U-h_{2}\right)^{p-1}\right) .
$$

Since $B / p B$ is regular local and any power of a complete intersection $B$-ideal is perfect, we are through. Therefore only the claims remain to be proved.

Set $\mathscr{Q}:=\mathscr{F}_{2}+(m)$. For claim (1), from (17) we only need to show

$$
s:=p^{-1} m\left(\omega-h_{1}\right)^{p-1}\left(\mu-h_{2}\right)^{p-1} \in \mathscr{Q} .
$$

Since $f g^{i} \in S^{p \wedge p^{2}}$, we get $a h_{2}^{p}+i b h_{1}^{p} \in p S$. Moreover, $\operatorname{grade}(Q)=3$ implies $a-q h_{1}^{p} \in p S$ and $b+i^{-1} q h_{2}^{p} \in p S$ for some $q \in S$. Write

$$
m=\left(\omega-h_{1}\right)\left(\mu^{i}-h_{2}^{i}\right)+h_{2}^{i}\left(\omega-h_{1}\right)+h_{1}\left(\mu^{i}-h_{2}^{i}\right) .
$$

and recall that $\left(\omega-h_{1}\right)^{p}=p\left(a-c_{1}^{\prime}\left(\omega-h_{1}\right)\right)$ and $\left(\mu-h_{2}\right)^{p}=p\left(b-c_{2}^{\prime}\left(\mu-h_{2}\right)\right)$. Then

$$
\begin{aligned}
s & \equiv p^{-1}\left[h_{2}^{i}\left(\omega-h_{1}\right)+h_{1}\left(\mu^{i}-h_{2}^{i}\right)\right]\left(\omega-h_{1}\right)^{p-1}\left(\mu-h_{2}\right)^{p-1} \bmod \mathscr{Q} \\
& \equiv a h_{2}^{i}\left(\mu-h_{2}\right)^{p-1}+b h_{1}\left(\mu^{i-1}+\cdots+h_{2}^{i-1}\right)\left(\omega-h_{1}\right)^{p-1} \bmod \mathscr{Q} \\
& \equiv a h_{2}^{i}\left(\mu-h_{2}\right)^{p-1}+i b h_{1} h_{2}^{i-1}\left(\omega-h_{1}\right)^{p-1} \bmod \mathscr{Q} \\
& \equiv q h_{1} h_{2}^{i}\left[h_{1}^{p-1}\left(\mu-h_{2}\right)^{p-1}-h_{2}^{p-1}\left(\omega-h_{1}\right)^{p-1}\right] \bmod \mathscr{Q} .
\end{aligned}
$$

Now $\left(\omega \mu^{i}-h_{1} h_{2}^{i}\right) \cdot P^{p-2} \subseteq \mathscr{Q},(19)$ and $P^{p} \subseteq \mathscr{Q}$ imply

$$
h_{2}^{i}\left(\omega-h_{1}\right)+h_{1}\left(\mu^{i}-h_{2}^{i}\right) \in\left(\mathscr{Q}:_{A} P^{p-2}\right) .
$$

Therefore for all $0 \leq j \leq p-2$

$$
\begin{aligned}
& \left(h_{2}^{i}\left(\omega-h_{1}\right)+h_{1}\left(\mu^{i}-h_{2}^{i}\right)\right) \cdot h_{2}^{p-2-j} h_{1}^{j}\left(\omega-h_{1}\right)^{p-2-j}\left(\mu-h_{2}\right)^{j} \\
= & h_{2}^{i-1}\left[\left(h_{2}\left(\omega-h_{1}\right)\right)^{p-j-1}\left(h_{1}\left(\mu-h_{2}\right)\right)^{j}\right]+ \\
& \left(h_{1}\left(\mu-h_{2}\right)\right)^{j+1}\left(\mu^{i-1}+\cdots+h_{2}^{i-1}\right)\left(h_{2}\left(\omega-h_{1}\right)\right)^{p-2-j} \in \mathscr{Q} .
\end{aligned}
$$

Thus

$$
h_{2}^{i-1}\left[\left(h_{2}\left(\omega-h_{1}\right)\right)^{p-j-1}\left(h_{1}\left(\mu-h_{2}\right)\right)^{j}\right] \equiv-i h_{2}^{i-1}\left(h_{1}\left(\mu-h_{2}\right)\right)^{j+1}\left(h_{2}\left(\omega-h_{1}\right)\right)^{p-2-j} \bmod \mathscr{Q} .
$$


It then follows that for any $1 \leq k \leq p-j-1$

$h_{2}^{i-1}\left[\left(h_{2}\left(\omega-h_{1}\right)\right)^{p-j-1}\left(h_{1}\left(\mu-h_{2}\right)\right)^{j}\right] \equiv(-i)^{k} h_{2}^{i-1}\left(h_{1}\left(\mu-h_{2}\right)\right)^{j+k}\left(h_{2}\left(\omega-h_{1}\right)\right)^{p-j-1-k} \bmod \mathscr{Q}$.

In particular for $j=0$ and $k=p-1$ we get

$$
h_{2}^{i-1}\left(h_{2}\left(\omega-h_{1}\right)\right)^{p-1} \equiv h_{2}^{i-1}\left(h_{1}\left(\mu-h_{2}\right)\right)^{p-1} \bmod \mathscr{Q} .
$$

Combining (21) and (20), we see that $s \in \mathscr{Q}$ and thus claim (1) holds.

To show one containment in claim $(2)$, note that $(p)+P^{p-1} \subseteq\left(\mathscr{F}_{2}:_{A} m\right)$ since $p+P^{p}=\mathscr{F}_{2}$. For the reverse inclusion, consider $y \in\left(\mathscr{F}_{2}:_{A} m\right)$. Lifting to $B$ and denoting lifts by $\sim$

$$
\tilde{y}\left(W U^{i}-h_{1} h_{2}^{i}\right) \in(p, F(W), G(U))+\left(W-h_{1}, U-h_{2}\right)^{p}=(p)+\left(W-h_{1}, U-h_{2}\right)^{p} .
$$

Using (19) we have $\tilde{y} \in\left(p,\left(W-h_{1}\right)^{p-1}, U-h_{2}\right)$. Similarly $\tilde{y} \in\left(p, W-h_{1},\left(U-h_{2}\right)^{p-1}\right)$ and from (7)

$$
\tilde{y} \in\left(p,\left(W-h_{1}\right)^{p-1},\left(U-h_{2}\right)^{p-1},\left(W-h_{1}\right)\left(U-h_{2}\right)\right) .
$$

Now assume for some $1 \leq i \leq 2^{-1}(p-1)-1$,

$$
\tilde{y} \in\left(p,\left(W-h_{1}\right)^{i}\left(U-h_{2}\right)^{i}\right)+\left(W-h_{1}, U-h_{2}\right)^{p-1} .
$$

Write $\tilde{y}=\alpha \cdot\left(W-h_{1}\right)^{i}\left(U-h_{2}\right)^{i}+\beta$ for some $\alpha \in B$ and $\beta \in(p)+\left(W-h_{1}, U-h_{2}\right)^{p-1}$. From (22):

$$
\alpha \cdot\left(W-h_{1}\right)^{i}\left(U-h_{2}\right)^{i}\left(W U^{i}-h_{1} h_{2}^{i}\right) \in(p)+\left(W-h_{1}, U-h_{2}\right)^{p} .
$$

Using the regular sequence $\left(p,\left(U-h_{2}\right)^{i+1},\left(W-h_{1}\right)^{i+1}, h_{2}^{i}\right) \subseteq B$, we get

$$
\alpha \in\left(p,\left(W-h_{1}\right)^{p-2 i-1}, U-h_{2}\right) .
$$

Similarly, using the regular sequence $\left(p,\left(W-h_{1}\right)^{i+1},\left(U-h_{2}\right)^{i+1}, h_{1}\left(U^{i-1}+\cdots+h_{2}^{i-1}\right)\right) \subseteq B$ we get $\alpha \in\left(p, W-h_{1},\left(U-h_{2}\right)^{p-2 i-1}\right)$. Thus by (7):

$$
\alpha \in\left(p,\left(W-h_{1}\right)^{p-2 i-1},\left(U-h_{2}\right)^{p-2 i-1},\left(W-h_{1}\right)\left(U-h_{2}\right)\right)
$$

and hence

$$
\tilde{y} \in\left(p,\left(W-h_{1}\right)^{i+1}\left(U-h_{2}\right)^{i+1}\right)+\left(W-h_{1}, U-h_{2}\right)^{p-1} .
$$

Thus starting from (23) we may induct on $i$ to get $\tilde{y} \in\left(p,\left(W-h_{1}\right)^{2^{-1}(p-1)}\left(U-h_{2}\right)^{2^{-1}(p-1)}\right)+\left(W-h_{1}, U-h_{2}\right)^{p-1}=(p)+\left(W-h_{1}, U-h_{2}\right)^{p-1}$. This shows $\left(\mathscr{F}_{2}:_{A} m\right)=(p)+P^{p-1}$ and all claims have been proved. Thus $R$ admits a birational MCM module. 
Remark 4.11. If $\operatorname{grade}(Q)=2$ and $p . d_{S}(S / Q)=3$ in the context of $4.10(3)$, we are not able to construct a birational MCM module over $R$ at present. However, if we allow an extension of the quotient field, then constructing an MCM module over $R$ may be possible in this case, see [KS21].

Remark 4.12. By a vector bundle on the punctured spectrum of a regular local ring $(S, \mathfrak{m})$ or simply a bundle on $S$ we mean a finitely generated reflexive $S$-module $M$ such that $M_{P}$ is $S_{P}$-free for all non maximal ideals $P \subseteq S$. One could use 4.10(2) to generate examples of non-trivial bundles $M$ on localizations of polynomial rings or power series rings over $\mathbb{Z}_{(p)}$ of dimension $d$ at least three such that $\operatorname{rank}_{S}(M)=p^{2}+d-3$. Moreover, these bundles would satisfy $p \cdot d \cdot S(M)=1$.

Let $d \geq 3$ and $(T, \mathfrak{n})$ be a $d$-dimensional unramified regular local ring of mixed characteristic $p$. Choose $(S, \mathfrak{m}) \subseteq T$ a three dimensional subring of $T$ that is an unramified regular local ring of mixed characteristic $p$ and a quotient of $T$ by a regular sequence (such a choice is possible for example when $T$ is a localization of a polynomial ring over $\left.\mathbb{Z}_{(p)}\right)$. Let $S \subseteq\left(E, \mathfrak{n}^{\prime}\right) \subseteq T$ be such that $E$ is regular local and $S=E /(t)$ for some $0 \neq t \in E$. Using 4.10(2), with the base ring as $S$, construct $R$ such that it is not $S$-free. Choose a minimal $S$-free resolution

$$
0 \longrightarrow S \stackrel{\psi^{T}}{\longrightarrow} S^{p^{2}+1} \longrightarrow 0
$$

Let $M^{\prime}$ be the cokernel of the $E$-matrix $\phi:=\left[\begin{array}{l|l}\psi^{T} & \mid t\end{array}\right]$

$$
0 \longrightarrow{ }^{\phi} E^{p^{2}+2} \longrightarrow M^{\prime} \longrightarrow 0
$$

so that p.d.E $(M)=1$. The ideal of maximal minors of $\psi^{T}$ is $\mathfrak{m}$-primary since $R$ is a bundle over $S$. Therefore the ideal of maximal minors of $\phi$ is $\mathfrak{n}^{\prime}$-primary and hence it is free on the punctured spectrum of $E$. Proceeding this way, we can construct a finite module $M$ over $T$ that is free on the punctured spectrum of $T$ and $p \cdot d_{\cdot T}(M)=1$. Moreover, since $M$ is an $S_{2} T$-module, it is $T$-reflexive (see [BH98] [Proposition 1.4.1] for example).

\section{Acknowledgement}

I would like to thank my Ph.D. advisor Prof. Daniel Katz for suggesting this problem and for his support through the course of this work. I would also like to thank the referee for a careful reading of the paper and suggestions for improvement.

\section{References}

[BH98] Winfried Bruns and H. Jürgen Herzog. Cohen-Macaulay Rings. Cambridge Studies in Advanced Mathematics. Cambridge University Press, 2 edition, 1998. 
[HK19] Craig Huneke and Daniel Katz. Uniform symbolic topologies in abelian extensions. Transactions of the American Mathematical Society, 372(3):1735-1750, May 2019.

[Hoc73] Melvin Hochster. Cohen-Macaulay modules. In James W. Brewer and Edgar A. Rutter, editors, Conference on Commutative Algebra, pages 120-152, Berlin, Heidelberg, 1973. Springer Berlin Heidelberg.

[HS06] Craig Huneke and Irena Swanson. Integral closure of ideals, rings, and modules, volume 13. Cambridge University Press, 2006.

[Kat99] Daniel Katz. On the existence of maximal Cohen-Macaulay modules over p-th root extensions. Proceedings of the American Mathematical Society, 127(9):2601-2609, 1999 .

[Kat21] Daniel Katz. Conductors in mixed characteristic. Journal of Algebra, 571:350375, 2021. Commutative Algebra and its Interactions with Algebraic Geometry: A volume in honor of Craig Huneke on the occasion of his 65th birthday.

[Koh86] Jee Koh. Degree p extensions of an unramified regular local ring of mixed characteristic p. Journal of Algebra, 99(2):310-323, 1986.

[KS21] Daniel Katz and Prashanth Sridhar. Small CM modules over repeated radical extensions in mixed characteristic. in preparation, 2021.

[KU97] Steven Kleiman and Bernd Ulrich. Gorenstein algebras, symmetric matrices, selflinked ideals, and symbolic powers. Transactions of the American Mathematical Society, 349(12):4973-5000, 1997.

[Rob80] Paul Roberts. Abelian extensions of regular local rings. Proceedings of the American Mathematical Society, 78(3):307-310, 1980.

[Rob12] Paul C Roberts. The homological conjectures. Progress in Commutative Algebra 1: Combinatorics and Homology, page 199, 2012.

[Sri21] Prashanth Sridhar. Existence of birational small CM modules over biquadratic extensions in mixed characteristic, 2021.

[Vas91] Wolmer V Vasconcelos. Computing the integral closure of an affine domain. Proceedings of the American Mathematical Society, 113(3):633-638, 1991. 